Para enlazar con este artículo / To link to this article:

http://dx.doi.org/10.14198/fem.2019.33.02

Para citar este artículo / To cite this article:

Gandarias Goikoetxea, Itziar, Montenegro Martínez, Marisela y Pujol Tarrés, Joan. «Interseccionalidad, identidad y articulación: hacia una política de la agregación». En Feminismo/s, 33 (junio 2019): 35-63. Dosier monográfico: Diálogos entre la democracia participativa y la interseccionalidad. Construyendo marcos para la justicia social, coords. Patricia Martínez-García y Jone Martínez-Palacios, DOI: 10.14198/fem.2019.33.02

\title{
INTERSECCIONALIDAD, IDENTIDAD Y ARTICULACIÓN: HACIA UNA POLÍTICA DE LA AGREGACIÓN
}

\section{INTERSECTIONALITY, IDENTITY AND ARTICULATION: TOWARDS AGGREGATION POLITICS}

Itziar GANDARIAS GOIKOETXEA

Universidad de Deusto itziar.gandarias@deusto.es http://orcid.org/0000-0003-4912-2941

\section{Marisela MONTENEGRO MARTÍNEZ}

Universitat Autònoma de Barcelona marisela.montenegro@uab.cat https://orcid.org/0000-0001-7382-9256

\section{Resumen}

Joan PUJOL TARRÉS

Universitat Autònoma de Barcelona joan.pujol@uab.cat http://orcid.org/0000-0002-0462-3278

El avance del neo-conservadurismo liberal amenaza las conquistas de los derechos sociales y civiles en un contexto de crisis económica. La incorporación de las políticas de identidad en los discursos conservadores, en tiempos de progresiva individualización y fragmentación social, dificulta la articulación de una respuesta emancipadora a las necesidades sociales de redistribución y reconocimiento. Esta contribución analiza el potencial de movilización y politización de la interseccionalidad como base articulatoria que reconoce las diferencias de posición. Partiendo del descentramiento de la identidad, el artículo explora tres figuras: ensamblajes, mundo zurdo, y política 
Interseccionalidad, identidad y articulación: hacia una política de la agregación

de lo múltiple. Estas figuraciones proporcionan metáforas articulatorias a la vez que reconocen simultáneamente diferencias, complejidad, singularidad y multiplicidad.

Palabras clave: interseccionalidad, políticas de identidad, articulación, figuraciones, desigualdades.

\begin{abstract}
The advancement of liberal neo-conservatism is threatening the conquests of social and civil rights in a context of economic crisis. The incorporation of identity-politics in conservative discourses, in times of progressive individualisation and social fragmentation, hinders the articulation of an emancipatory response to the social needs of redistribution and recognition. This contribution analyses the mobilising and politicising potential of intersectionality as an articulatory foundation that recognises differences. Starting from the decentering of identity, the paper explores three figurations: assemblages, mundo zurdo, and the politics of the multiple. These figurations provide articulatory metaphors while recognising differences, complexity, uniqueness and multiplicity.
\end{abstract}

Keywords: Intersectionality, identity politics, articulation, figurations, inequalities.

\title{
1. INTRODUCCIÓN
}

¿Ustedes saben lo que son las TERF? No se sientan ignorantes, yo tampoco lo sabía hasta hace dos días. TERF, acrónimo de Trans Exclusionary Radical Feminist, resulta que somos nosotras. Sí, las feministas de siempre, las que reclamamos desde hace 200 años libertad, igualdad, solidaridad. Ese término se lo han inventado un grupo de transexuales, apoyadas al parecer por otro grupo de LGTB, que siguiendo la teoría queer -aquella que dice que no nacemos con una predeterminación de sexo sino que a lo largo de la vida escogemos variablemente el que queremos- han decidido que ni el sexo, ni la edad, pueden ser definitorios. (Falcón, 2017)

Desgraciadamente la interseccionalidad sigue siendo una palabra que el feminismo hegemónico vacía de contenido cuando la tiene que poner en práctica. Hay un gran número de periodistas, cadenas de televisión y periódicos que están haciendo un gran esfuerzo por la huelga ¿Cuántos de esos medios tienen personas negras trabajando? ¿Cuántas de esas periodistas, casi en su totalidad blancas, se han acordado de las mujeres racializadas? En una esfera tan blanca es poco probable vernos representadas.

(Blog Afroféminas, 2018)

Feminismo/s 33, junio 2019, pp. 35-63 
Interseccionalidad, identidad y articulación: hacia una política de la agregación

Dadas las condiciones crecientes de precariedad de la vida en todas sus formas, humanas y ecológicas, es sorprendente, como sugiere Fraser (2), que una «nueva coalición de 'New Dealers': sindicalistas, personas sin empleo o con empleos precarios, feministas, ecologistas y antiimperialistas; social demócratas y socialistas democráticos» no haya podido producir un proyecto contrahegemónico que proteja a la sociedad y a la naturaleza del neoliberalismo. Una respuesta apresurada a esta pregunta podría apuntar al éxito de las políticas de identidad inspiradas por los nuevos movimientos sociales de los años 60 y que muestran signos de agotamiento a fines de 2010. Bajo esta narrativa, la posición de los grupos sociales en la sociedad depende de las características de este grupo social (raza, género, sexualidad, capacidades, etc), una posición que conduce a formas específicas de opresión. Las políticas de igualdad no reconocen las especificidades de cada ubicación social y el valor de dicha ubicación, por lo que los grupos sociales deben luchar por políticas de reconocimiento que conduzcan al respeto y a la protección de tales formas de vida. La conciencia social proveniente de la desmitificación de la experiencia única y compartida de opresión, derivada de la pertenencia a un grupo social particular, constituye un fuerte impulso para la transformación social. La dificultad surge, sin embargo, en cómo articular diferentes experiencias únicas de opresión, algo inherente al surgimiento de políticas de identidad (Breines 19-51).

Una primera respuesta sugeriría que la dificultad de consolidar articulaciones que trasciendan la desconfianza ante la imposibilidad de comprensión de nuestra experiencia de opresión está coronando a gobiernos autoritarios racistas, sexistas y homófobos. Después de años de fuerte crisis económica en España, el partido político Vox obtiene un importante resultado electoral en las últimas elecciones en Andalucía, incluyendo en su programa electoral propuestas xenófobas ( «posibilidad de perder la nacionalidad adquirida por actividades contra la soberanía, seguridad o independencia nacional», acción 20), sexistas («supresión de organismos feministas radicales subvencionados, persecución efectiva de denuncias falsas», acción 70), y homófobas («promulgación de una ley orgánica de protección de la familia natural que la reconozca como institución anterior al Estado», acción 71). Esta no es una tendencia aislada, dado el clima político internacional donde las políticas de identidad son usadas por posturas de derecha reaccionaria. Por ejemplo,

Feminismo/s 33, junio 2019, pp. 35-63 
Interseccionalidad, identidad y articulación: hacia una política de la agregación

Marine Le Pen hace una interpelación velada a la identidad mayoritaria en términos de «sois los olvidados, sois la mayoría invisible» (Marine Le Pen, Discurso en Metz, 10 de diciembre de 2011, citado en Ivaldi 231), o Donald Trump en términos de «los hombres y mujeres olvidados de nuestro país, ya no serán olvidados» (New York Times). Por su parte, Jair Bolsonaro declara en su discurso de toma de posesión: «vamos a unir al pueblo, valorizar la familia, respetar las religiones y nuestra tradición judeo-cristiana, combatir la ideología de género, conservando nuestros valores. Brasil volverá a ser un país libre de las amarras ideológicas» (Bolsonaro), mientras Damares Alves, la nueva ministra de familia, señala que «es una nueva era para Brasil: el niño viste de azul y la niña viste de rosa» (Brasilia agencias).

Señalan Hardt y Negri, respecto de la reestructuración de la producción fordista a la post-fordista, que «si no hubiera habido revueltas estudiantiles y obreras en los '60, si no hubiese ocurrido el 1968 y la segunda ola de movimientos feministas, si no hubiese estado toda la serie de luchas anti-imperialistas, el capital se habría conformado con mantener su propio dispositivo de poder» (241). Siguiendo esta idea, ¿nos encontramos en un proceso de reestructuración de los mecanismos de poder debido al éxito de las políticas de la identidad? ¿Son las políticas de identidad de Trump, Le Pen o Bolsonaro una coaptación conservadora del motor de los nuevos movimientos sociales? Nancy Fraser compara la gravedad de la situación actual a la Gran Depresión de los años 1930; la actual inacción ante la progresiva precarización de la vida, a la histórica respuesta ante la desregulación del mercado a través del New Deal en lo que viene a llamarse «doble movimiento» (Polanyi). Fraser (189-209) recurre a tres factores para explicar la falta de respuesta articulada y contundente a la situación actual: (1) falta de un atrevido liderazgo político que implemente las necesarias medidas de cambio; (2) el debilitamiento de la clase trabajadora y de los sindicatos por la transformación de la producción industrial fordista a la dominancia post-fordista del sector financiero y la progresiva fragilidad de los estados como agentes de redistribución por el cambio geopolítico desencadenado por el óbito de los acuerdos de Bretton Woods en 1971. Fraser (209-227), reconociendo la importancia de estos factores, considera que el análisis (1) no puede reducirse a la psicología de líderes políticos; (2) ha de tener en consideración las importantes movilizaciones basadas en el reconocimiento y la reproducción social (género, sexualidad, 
Interseccionalidad, identidad y articulación: hacia una política de la agregación

o nacionalidad); y (3) debe reconocer la existencia de entidades supranacionales con suficiente fortaleza geopolítica. Fraser (227-243) concluye que la imposibilidad de articular una respuesta estriba en la dificultad de integrar la fuerza emancipadora de los nuevos movimientos sociales en la oposición entre mercantilización y protección social, en lo que debería constituir un triple movimiento. Sin embargo, los actuales y exitosos movimientos emancipatorios centrados en las políticas de identidad siguen una lógica de individualización que ha trasladado el foco de análisis desde la transformación de las estructuras sociales que producen discriminación y exclusión social en términos de desigualdad e injusticia, hacia una localización psicológica de estas estructuras en términos de «prejuicio» y «ofensa». La psicologización del análisis de la temática del racismo o el sexismo genera políticas que pivotan en torno a cuestiones taxonómicas y de corrección política en lugar de una transformación social efectiva.

En este clima político adverso, encontramos crecientes tensiones dentro y entre círculos feministas, antirracistas y LGBTI. Conflictos que generan una profunda ansiedad ante el avance global de pensamiento y políticas neoconservadoras en un momento en que las perspectivas interseccionales son ampliamente aceptadas en los movimientos antirracistas, feministas y LGBTI del Estado español, perspectivas incluidas en políticas públicas que se desarrollan en el País Vasco y Cataluña (Unzueta 145-166; Coll-Planas y Cruells 153-172). Esta ansiedad nos hace preguntarnos si, quizá, es necesario reformular la política de identidad para mantener políticas progresivas que enfaticen agregación frente a diferenciación, dado que «el avance de la conciencia de identidad liberal ha marcado un retroceso en la conciencia política liberal» (Lilla 10). Podríamos, por otra parte, leer estos conflictos como la necesidad de un retorno a la esencia de las políticas de identidad y de profundizar nuestro análisis y práctica intersectoriales en un reconocimiento de que «la única forma de salvar el cuerpo político es reconocer que las políticas de la identidad son parte de la solución, no del problema» (Walters 483). Quizá sea necesario producir nuevas figuraciones feministas que transformen nuestra comprensión de la práctica feminista.

Este artículo tiene como objetivo analizar en qué medida la interseccionalidad puede ser una herramienta para la movilización y repolitización en los tiempos actuales de crisis y para la articulación de movimientos sociales

Feminismo/s 33, junio 2019, pp. 35-63 
Interseccionalidad, identidad y articulación: hacia una política de la agregación

en base a la enunciación de las diferencias como elemento fundante y punto de arranque. Para ello, en primer lugar, desarrollamos las principales críticas a las políticas de identidad, las potencialidades de la interseccionalidad situada y contingente y los usos que vacían su carácter político. A continuación, desarrollamos tres figuraciones: los Ensamblajes, el Mundo Zurdo y la Política de lo Múltiple, que rompen con el binarismo tradicional de la lógica identitaria y que nos ofrecen nuevas construcciones - mundos a partir de la frontera, la diferencia y la multiplicidad. Finalizamos con algunas consideraciones útiles para seguir pensando y configurando articulaciones más allá de la lógica identitaria.

\section{INTERSECCIONALIDAD: IDENTIDADES, PODER Y POLITIZACIÓN}

La alusión a colectivos «privilegiados por las políticas públicas» o al «olvido de la mayoría blanca» en los discursos de líderes políticos actuales da cuenta de hasta qué punto los movimientos identitarios han sido decisivos en el devenir político de nuestra contemporaneidad. La premisa de una experiencia compartida por los miembros de cierto grupo social ha funcionado como poderoso lugar de enunciación para denunciar determinadas formas de discriminación, exclusión y violencia, así como para demandar derechos colectivos. Demandas que en ocasiones han recibido respuestas institucionales; las cuales, a su vez, han contribuido a definir qué colectivos se considerarán «grupos minoritarios» a los que es preciso atender. Esta dinámica coloca a dichos grupos en el foco de las medidas de protección, supervisión y control desplegadas a través de las políticas públicas (Epstein 9-54; Spade 1031-1055).

Desde perspectivas construccionistas, de estudios culturales y feministas ha habido un amplio interés por teorizar las formas en las que se constituyen las identidades que sirven de base para la participación política. Siguiendo a Stuart Hall (1-17), en los procesos de construcción identitaria, la identidad constituye sus límites en relación con aquello que no es; esto es, por virtud de la construcción de la diferencia, que funcionará como su exterior constitutivo. Identidad y diferencia no corresponden a atributos naturales o esenciales de los sujetos, sino que emergen en procesos sociohistóricos atravesados por prácticas institucionales y discursos económicos, culturales y políticos (Brah). 
Interseccionalidad, identidad y articulación: hacia una política de la agregación

De manera que la base sobre la que se asientan los movimientos identitarios corresponde a una zona de tensión e inestabilidad que proviene de las redes de poder y resistencia que circulan entre los polos de la identidad y la diferencia (Hall 4). La inestabilidad inherente a toda formación identitaria implica que la conformación de una categoría identitaria, como por ejemplo la de «mujer», responde a mecanismos sociohistóricos de inclusión y exclusión, imbuidos en complejos entramados de poder frecuentemente naturalizados (Romero Bachiller 117).

Algunas de las críticas a las políticas de la identidad residen precisamente en los efectos de naturalización que se desprenden de la lógica identitaria. La esencialización, al considerar la identidad como algo que se «es» y que es inmutable; la homogeneización; al entender que los miembros de la categoría sean considerados idénticos entre sí; y la normalización, que implica la regulación de cuerpos y prácticas, contribuyen a la reificación de las categorías identitarias. Y es a través de este conjunto de procesos que se reiteran sistemáticamente las diferencias y asimetrías, como, por ejemplo, las relativas al binarismo de género, que estructuran la propia discriminación (Romero Bachiller y Montenegro 8). Así, la configuración identitaria de las luchas políticas comporta la inherente dificultad de dar cuenta de la gran variabilidad que puede haber en las propias categorías identitarias. Y de este modo contribuye a la invisibilización de las posiciones minorizadas, pues, aunque las reivindicaciones corresponden a demandas particulares, son formuladas «en nombre de» todas las personas que pertenecerían a determinada categoría.

En el contexto de estos debates, autoras como Jennifer Nash (1-15) consideran el concepto de interseccionalidad como una herramienta útil para responder a las críticas a las políticas de la identidad: cuestiona la homogeneización de las categorías identitarias y visibiliza la multiplicidad de los ejes de opresión en el seno de una determinada configuración identitaria (Platero 135-172). Aunque Kimberlé Crenshaw (Demarginalizing 139-167) es conocida como quien acuña el concepto de interseccionalidad, ya en 1851 Sojourner Truth (463-464) con su famoso discurso «Ain't I a Woman» pronunciado en un encuentro activista en Ohio, estableció las bases para la deconstrucción de la noción de «mujer» que era predominante en su contexto (Collins, Black Feminist). El Manifiesto del Colectivo Combahee River «A Black Feminist Statement», escrito en 1977, también es frecuentemente

Feminismo/s 33, junio 2019, pp. 35-63 
Interseccionalidad, identidad y articulación: hacia una política de la agregación

referenciado como parte de esta tradición crítica, considerándolo uno de los documentos fundacionales del feminismo negro contemporáneo. En el Manifiesto el colectivo no sólo expresa el interés político por diferentes sistemas de opresión, sino que, además, postula ya el carácter interconectado de los mismos (Gumbs 142-152). Incluso en el Combahee River Collective, a pesar de su agenda radical, «tuvieron problemas para dar cuenta de la clase social [...] una cuestión que causó división y a la que se esperaba que se restara importancia en nombre de la unidad racial» (Breines 139). ¿Significa esto que la política de identidad está reñida con la política articulatoria? Vamos a volver a leer cómo la Declaración colectiva de Combahee River aborda las políticas de identidad:

Nos damos cuenta que las personas que se preocupan realmente por nosotras y que trabajan continuamente por nuestra liberación somos nosotras mismas. Nuestras políticas surgen de un amor saludable por nosotras mismas, nuestras hermanas y nuestra comunidad, el cual nos permite continuar con nuestra lucha y nuestro trabajo. Este interés sobre nuestra propia opresión se enraíza en el concepto de políticas identitarias. Creemos que las políticas más profundas y potencialmente más radicales surgen directamente de nuestra propia identidad, como una postura que se niega a trabajar para terminar con la opresión de otras personas. (Combahee River Collective 78-79)

Considerando el contexto del manifiesto, la declaración denunció la falta de atención a múltiples ejes de opresión por parte de aliados cercanos como los movimientos antirracistas y feministas. En lugar de un llamado a la desarticulación, la declaración asegura la colaboración con grupos antirracistas y feministas en un momento en que la comunidad negra se mostró reticente a aceptar las demandas feministas y el movimiento feminista no prestó atención a los temas raciales. La declaración es un compromiso de articulación con posiciones que rechazan sus demandas y, debido a esto: «si las mujeres Negras fueran libres, significaría que todas las demás personas tendrían que ser libres también, ya que nuestra libertad necesitaría de la destrucción de todos los sistemas de opresión» (Combahee River Collective 82). Toda la declaración no argumenta solo luchar "por nuestra lucha». Se propone abordar diferentes formas de opresión, opresiones que están particularmente encarnadas en las mujeres negras. Como sugiere Keeanga-Yamahtta Taylor, «las mujeres del CRC no definieron las 'políticas de la identidad' como excluyentes, que 
Interseccionalidad, identidad y articulación: hacia una política de la agregación

implicaría que solamente aquellas que experimentan una opresión particular podrían luchar contra ésta» (Taylor 11). Sin embargo, la atención a ciertos aspectos del CRC ha reformulado la solidaridad y articulación de las formulaciones iniciales de las políticas de identidad en términos de lobbying liberal donde cada posición solo apoya sus propios problemas; un replanteamiento que justifica políticas de derecha que aluden a la «blancura», defendiéndose de la «herida blanca».

Así mismo, publicaciones de principios y mediados de los 80 como This bridge called my Back: writings by radical women of color (Moraga y Anzaldúa), Sister Outsider: Essays and speeches (Lorde), Under western eyes: feminist scholarship and colonial discourses (Mohanty 61-88), entre otras, también contribuyeron a la teoría feminista antirracista en la que se inscribe la noción de interseccionalidad (Carastathis 306).

Crenshaw (Demarginalizing 139-167, Mapping the Margins 1241-1299) se inserta en estas tradiciones y desde allí desarrolla la noción de interseccionalidad, con la cual subraya la necesidad de tomar en cuenta las dimensiones múltiples y simultáneas de las diferentes desigualdades tanto en el ámbito del Derecho como en la movilización política. Para esta autora, focalizar en un solo eje de discriminación (como el género o la raza) tiene como efecto que se borra a las mujeres negras de la conceptualización, identificación y solución de la discriminación por raza y sexo, pues se limita la indagación a las experiencias de los miembros privilegiados de cada grupo (mujeres blancas / hombres negros). Con este trabajo, Crenshaw contribuye a la visibilización de los mecanismos de exclusión que operan sobre aquellas posiciones que no concuerdan con la uniformidad que recogen las políticas de identidad. La noción de interseccionalidad se va conformando así como una herramienta útil para introducir las interrelaciones de género, etnicidad y clase -entre otros ejes de opresión- en los análisis feministas, así como para estudiar las formas en las que las relaciones de poder configuran posiciones subordinadas dentro de las lógicas de los movimientos identitarios (Anthias y Yuval-Davis 62-75; Davis 65-85).

Nira Yuval-Davis (3) apunta precisamente a esta cuestión. Según ella, aunque el concepto se desarrolló originalmente como contrario a las políticas de la identidad, algunas de las perspectivas que trabajan la interseccionalidad se han transformado en una suerte de "política de la identidad fragmentada», 
Interseccionalidad, identidad y articulación: hacia una política de la agregación

donde el foco ya no sería «mujeres» o «negros», sino «mujeres negras». De manera que se reproduce el efecto de homogeneización de las políticas de la identidad, pero esta vez, a través de la «subcategoría» que surge del cruce de diferentes ejes de subordinación. Como consecuencia, se tomarían por iguales las experiencias de, por ejemplo, todas las «mujeres migradas», invisibilizando la heterogeneidad de esta «nueva» categoría (Gandarias 73-93). De hecho, la lógica de sub-categorías identitarias podría irse reproduciendo, añadiendo nuevos ejes de opresión y generando nuevas categorías como, por ejemplo, las «mujeres migrantes lesbianas»: un producto de la sumatoria de opresiones (Hancock 63-79). El exterior constitutivo de dicha identidad correspondería a aquellas posiciones «no marcadas» («no interseccionales»), contribuyendo, así, a una suerte de «binarismo identitario» que distinguiría entre posiciones «vulnerables» (marcadas) y «privilegiadas» (no marcadas). La preeminencia de focalizar y analizar sujetos marcados por múltiples opresiones en los estudios con perspectiva interseccional impide visualizar, como dice Yuval-Davis (1-10), aquello que los estudios críticos de la raza y la etnicidad han señalado: que no sólo las personas negras están racializadas y que los varones también tienen género. Jasbir Puar hace una crítica similar:

Mi punto aquí es simple: la interseccionalidad rara vez se refiere al trabajo con sujetos blancos, con la importante excepción de mujeres blancas de clase trabajadora. Y, de manera más general, rara vez se refiere al trabajo relacionado con sujetos privilegiados, como, por ejemplo, los hombres blancos de clase alta. Si bien el estudio de estos sujetos puede efectivamente involucrar un análisis o enfoque interseccional, la referencia al término en sí está suturada a un referente que conduce a la esencialización racial. (I Would Rather 64)

La tendencia identificada por estas autoras en relación con la hipervisibilización de ciertos grupos en los estudios de interseccionalidad, puede ser leída en términos de la reiteración de prácticas que contribuyen a la constitución de identidades. Esto es, la tendencia que identifican las autoras muestra una operación de diferenciación y asimetría que contribuye a señalar / identificar a aquellos sujetos marcados por diversas opresiones. Una lógica que guarda cierta coherencia con los procesos de diferenciación entre posiciones normativas y no normativas como los que identifica la propia Puar (I Would Rather 49-66) cuando critica la homologación de la interseccionalidad con la 
Interseccionalidad, identidad y articulación: hacia una política de la agregación

«gestión de la diversidad». Esta gestión, lejos de referir a prácticas politizadas, refiere a una suerte de «llamado a la inclusión» dentro de las categorías convencionales existentes. O, como afirma Mohanty, una forma de entender la interseccionalidad como una «variación benigna» (On race and voice 181), lo cual sustrae al concepto de toda implicación de cambio. Esto resulta también compatible con un contexto de multiculturalidad neoliberal como el contemporáneo. Ya que, como afirma Patricia Hill Collins (The difference 19-39), las promesas de inclusión y de libertad personal de las democracias liberales apuntan a los derechos individuales de ciudadanía como la piedra angular de la política democrática.

Aunque las críticas que realizan las autoras se relacionan con la falta de potencial político del uso de la interseccionalidad como herramienta para la gestión de la diversidad, consideramos que precisamente ese uso que se da a la noción de interseccionalidad -o a términos parecidos- es uno de los que debe ser objeto de escrutinio. Es decir, indagar sobre las maneras en las que se concreta el mainstreaming de la interseccionalidad en diferentes estamentos institucionales provee de un espacio privilegiado para comprender el funcionamiento de las dinámicas de definición y delimitación de problemas, sujetos y programas de acción en determinada área (Nayak, Montenegro y Pujol 245). El estudio de las políticas públicas es fundamental para Collins (The difference 19-39), pues corresponde a uno de los espacios donde se ejerce el «dominio estructural del poder».

En el análisis que Carmen Romero Bachiller y Marisela Montenegro (1-10) realizaron a las llamadas leyes contra la LGTBIfobia ${ }^{1}$ de Barcelona y Madrid desde una perspectiva interseccional, observaron los procesos de identificación y diferencia que se materializan en los textos de las leyes. Por un lado, estaban claramente identificadas las categorías centrales empleadas (correspondientes a las diferentes letras del acrónimo LGTBI). Y, por otro, se definían «discriminaciones múltiples» (no aparece en ninguna de las dos leyes la noción de interseccionalidad). Aunque apreciaron ciertas diferencias

1. «Ley 11/2014, del 10 de octubre, para garantizar los derechos de Lesbianas, Gays, Bisexuales, Transgéneros e Intersexuales y para erradicar la Homofobia, la Bifobia y la Transfobia», y «Ley 3/2016, de 22 de julio, de Protección Integral contra la LGTBIfobia y la Discriminación por Razón de Orientación e Identidad Sexual en la Comunidad de Madrid». 
Interseccionalidad, identidad y articulación: hacia una política de la agregación

entre los dos documentos, identificaron una lógica común subyacente: la categoría central en los dos casos es la de orientación sexual o identidad de género, siendo la «otra» discriminación entendida como subsidiaria. Al listar las categorías identitarias que podrían intersectar con las principales, las leyes refieren a la «pertenencia» a dicha categoría, observándose un uso de una lógica identitaria (como migrante, persona con discapacidad...) para el abordaje de las «discriminaciones múltiples». Así, la manera en las que se presentan las «otras» categorías de discriminación, «refuerza la lógica sumatoria que universaliza las categorías, de modo que estas aparecen como compartimentos estancos y al tiempo isomórficos, que añaden aditivamente otra carga más a las personas que ocuparan ambas categorías» (Romero Bachiller y Montenegro 9).

Este ejemplo ilustra la preeminencia de la lógica identitaria en este marco legal. La preeminencia de la identidad tanto en trabajos académicos como en las políticas públicas apunta a que el descentramiento de la perspectiva identitaria del concepto de interseccionalidad implica un reto teórico, metodológico y político. Aun así, hay diversas aproximaciones al concepto que no están abocadas al análisis de la posición del sujeto, sino que atienden a diferentes aspectos de los contextos y de las dinámicas de poder involucradas en la generación de subordinaciones y privilegios. Yuval-Davis (1-10) desarrolla las herramientas teóricas y metodológicas para una interseccionalidad situada. Esta presta especial atención a los contextos de interacción con el fin de comprender las formas en las que se distribuye el poder. El propósito es identificar y analizar las diferentes ubicaciones y jerarquías que emergen de las redes de poder de la sociedad. Suryia Nayak y Rachel Robbins (1-5) también proponen entender la interseccionalidad como situada, aunque no exactamente en la misma línea que Yuval-Davis (1-10). Para las autoras, no es posible entender la interseccionalidad sin un análisis riguroso y localizado de las relaciones entre contexto, experiencia, práctica y producción de conocimiento. La interseccionalidad situada para ellas es aquella que entiende que diferencias particulares en contextos específicos producirán relaciones particulares de discriminación y subalternización. Por su parte, Patricia Hill Collins (The difference 19-39) propone un modelo de análisis en tres niveles (matriz de dominación, marcos de dominio específicos y vida cotidiana en

Feminismo/s 33, junio 2019, pp. 35-63 
Interseccionalidad, identidad y articulación: hacia una política de la agregación

la comunidad) en el que también se cuestionan las lógicas de posiciones e identidades «marcadas» identificadas en la literatura.

Estas perspectivas permiten focalizar el análisis en las formas en las que se construyen y mutan las propias categorías; entendidas estas como resultados de ordenamientos concretos asentados en prácticas y discursos institucionales y cotidianos. El descentrar la mirada de la posición del sujeto, alrededor del cual se definen políticas e intervenciones, genera una oportunidad para nuevos cuestionamientos. También permite virar el foco hacia los mecanismos que dibujan -reiterada, pero no inexorablemente- las dinámicas de diferenciación y asimetría que nos afectan (Nayak, Montenegro y Pujol 230-250). Este cambio de perspectiva, que desvía el haz de luz que ilumina a «los sujetos», para alumbrar a las «imperfecciones del sistema» también puede contribuir a contravenir las lógicas particularistas, individualistas y fragmentadas que se desprenden del foco en la identidad. Metodológicamente esto implica analizar los contextos concretos, situados histórica y geográficamente, en los que las dinámicas de diferenciación tienen lugar, con el fin de comprender cómo se configuran las continuidades y discontinuidades de las dinámicas de poder. De esta manera se busca dar cuenta de la contingencia inherente a todo cierre categorial que emerge de los procesos de diferenciación y, al mismo tiempo, de los procesos de reproducción de espacios de subordinación (Romero Bachiller y Montenegro 12). Esto no significa un «olvido» de los sistemas de opresión sedimentados por la recurrencia de las relaciones de poder que los constituyen, sino más bien subraya la necesidad de atender también al carácter abierto y transformable de los criterios y mecanismos de diferenciación y asimetría. En este sentido, Romero Bachiller y Montenegro (12) afirman:

La distribución de vulnerabilidades a partir de los procesos de diferenciación responde a prácticas sistemáticas y reiteradas de diferenciación y asimetrías que se actualizan en situaciones concretas en las que determinadas diferencias emergen como más significativas que otras. Si la sistematicidad se explica por la recurrencia, la contingencia asegura el carácter abierto y potencialmente transformable de los criterios de diferenciación que son actualizados / actualizables en momentos sociohistóricos concretos que los vuelven significativos.

Esta aproximación, entonces, nos invita a reconocer y actuar comprendiendo los espacios como potencialmente abiertos a la politización y la creación de 
Interseccionalidad, identidad y articulación: hacia una política de la agregación

nuevas -e inesperadas- configuraciones de poder. Se trata de un análisis que deja de ver a la posición para mirar el acontecimiento y que se despliega en lugares y situaciones concretas. Desde este marco, proyectar la profundización democrática pasaría, por una parte, por cuestionar y erosionar los mecanismos sedimentados de participación política que pivotan sobre la idea de la necesidad de similitud entre participantes -en términos de características, intereses, recursos...- y, por otra, por generar espacios insospechados de articulación a partir del reconocimiento de la multiplicidad de las diferencias. Con este extracto, nos hacemos eco de las palabras que Audre Lorde pronunció en la Conferencia Nacional Gay y Lésbica del Tercer Mundo en 1979:

También estamos aquí para examinar nuestros roles como poderosas fuerzas dentro de nuestras comunidades. Porque ninguno/a de nosotros/as será libre hasta que todos/as seamos libres, y hasta que todos los miembros de nuestras comunidades sean libres. Así que estamos aquí para ayudar a formar un mundo donde todas las personas puedan florecer, más allá del sexismo, más allá del racismo, más allá del edaìsmo, más allá del clasismo y más allá de la homofobia. Para hacer esto, debemos vernos dentro del contexto de una civilización que tiene notoria falta de respeto y aversión por cualquier valor humano, por cualquier creatividad humana o diferencia humana genuina. $\mathrm{Y}$ es sobre nuestra capacidad para mirar honestamente nuestras diferencias, de verlas como creativas en vez de divisivas, que nuestro futuro éxito puede residir. (Lorde 209)

\section{FIGURACIONES PROMETEDORAS}

Frente a los riesgos que se desprenden de las aproximaciones identitarias -incluyendo algunas lecturas de la interseccionalidad que hemos señalado anteriormente-, proponemos indagar en nuevas figuraciones que descentren el peso de la identidad y recuperen el potencial político de la interseccionalidad. Para ello, exploraremos tres figuraciones: «Ensamblajes» (Puar), «Mundo Zurdo» (Anzaldúa) y «La Política de lo Múltiple» (Castillo).

\subsection{Los ensamblajes de Jasbir Puar}

Si las perspectivas identitarias están atravesadas por la metáfora del grupo social, la lectura identitaria de la perspectiva interseccional produce una multiplicidad estratificada de agrupaciones caracterizadas por atributos 
Interseccionalidad, identidad y articulación: hacia una política de la agregación

esenciales. Cada estrato puede segmentarse infinitesimalmente para producir una identidad experiencial inconmensurable e incomprensible para alguien situada en un estrato distinto; posiciones esencialmente inconmensurables que imposibilitan la articulación. ¿Es posible una relectura post-identitaria de la interseccionalidad que permita enlazar posiciones diversas sin caer en homogeneizaciones categoriales?

El concepto de «agenciamiento», del verbo agencer (organizar, diseñar, juntar), enfatiza las relaciones y patrones de relación entre un conjunto de elementos heterogéneos (Deleuze y Guattari; Puar, I Would Rather 49-66). Jasbir Puar incorpora esta acepción en su propuesta de ensamblajes, elaborando una figuración que conceptualiza las posiciones sociales en términos de multiplicidad (frente la unidad) y acontecimiento (frente la esencia). Tratemos estos dos puntos en detalle.

La unidad emerge de una serie de relaciones intrínsecas entre partes diferenciadas para formar un todo orgánico (Nail 21-37), una metáfora organicista que establece una doble diferenciación entre sus elementos jerarquizados y entre los otros organismos. A diferencia de los organismos, los ensamblajes se definen por relaciones de composición, mezcla y agregación; constituyen una multiplicidad sin partes claramente diferenciadas ni un todo unificado. Dar importancia a la relación entre elementos heterogéneos permite que el ensamblaje agregue, reste o recombine elementos sin crear o destruir una entidad esencial, produciendo un «todo fragmentario» (Deleuze y Guattari 16) donde los elementos no son imprescindibles como si fueran «piezas de un rompecabezas» (Deleuze y Guattari 23). El ensamblaje es múltiple, en tanto que cada recombinación de elementos produce un nuevo ensamblaje abierto a la combinación y al cambio de consistencia, donde lo relevante no son los elementos que forman parte del ensamblaje sino el conjunto de relaciones que lo conforman (Deleuze y Parnet, 8).

Por otra parte, el ensamblaje se caracteriza por el acontecimiento (responde a las preguntas de cómo, dónde, cuándo) en lugar de la esencia (qué es). En lugar de una entidad orgánica esencialmente distinta a otras, los ensamblajes están caracterizados por aspectos contingentes. Una lectura en términos de ensamblaje asume que las posiciones sociales son un proceso dentro de un entramado de eventos sociales e históricos, y la aparente estabilidad de una determinada posición social es el resultado de un accidente, un

Feminismo/s 33, junio 2019, pp. 35-63 
Interseccionalidad, identidad y articulación: hacia una política de la agregación

acontecimiento (Deleuze y Guattari 10); una perspectiva que permite pensar fuera de las lógicas identitarias esencialistas (Nail 21-37).

Corremos el peligro de que la traducción de «acontecimiento» por «ensamblaje» debilite la dimensión dinámica y relacional frente a la estática y substantiva (Law 41), peligro que reconoce Jasbir Puar señalando el análisis que realiza John Phillips al respecto y preguntándose sobre los efectos productivos de esta dificultad de traducción (I Would Rather 57). Sin embargo, usa la lógica del ensamblaje para situar el interés de la interrogación en las posibilidades pragmáticas en lugar de la pregunta esencialista sobre «qué son los ensamblajes». Esta línea permite poner en diálogo las nociones de interseccionalidad y ensamblaje para construir una conversación fructífera entre las teorías feministas sobre interseccionalidad y pos-representativa o post-humana: «Las identidades interseccionales son subproductos de los intentos de calmar y sofocar el movimiento continuo de los ensamblajes, capturarlos y reducirlos, aprovechando su movilidad amenazadora» (Puar, Terrorist Assamblages 213). Considera que son dos perspectivas en fricción vibrante en las que, si las tomamos como opuestas, encontramos que la interseccionalidad considera sus aspectos estáticos e invariantes (raza, sexo, discapacidad...) mientras que los ensamblajes se centran en categorías contextualmente salientes como resultado de un encuentro particular y temporalmente mutable. Por otra parte, en tanto que complementarias, la interseccionalidad se centra en la cristalización del ensamblaje en un determinado cronotopo, siendo necesario reconocer el carácter socio-histórico y contextual de esta particular cristalización. El análisis interseccional de una posición social es, de este modo, un análisis contextual que no puede dar cuenta de la indeterminación histórica de las múltiples posibles subjetivaciones. En otras palabras, las experiencias de las mujeres blancas lesbianas exceden a la localización interseccional en términos de «mujer blanca lesbiana», una localización interseccional que varía en función del contexto socio-histórico. Al mismo tiempo, las personas tienen una experiencia múltiple y contextual de la posición social, pero eso no significa que «pertenezcan» a esa posición social. Por ejemplo, una «mujer española blanca» se convierte en «latina» en Estados Unidos, de modo que la «blanquitud»o «latinidad»son el producto de ensamblajes racializadores que generan posiciones interseccionales en función del contexto social. Una determinada posición interseccional no precede al movimiento, sino que

Feminismo/s 33, junio 2019, pp. 35-63 
Interseccionalidad, identidad y articulación: hacia una política de la agregación

forma parte del movimiento (Puar, Terrorist assemblages), siendo habitual que la complejidad del proceso (como, por ejemplo, racialización) se confunda con el producto (raza) debido a la predominancia de la representación en la transformación política. Mientras que la interseccionalidad pone el foco en las prácticas y productos institucionales, el ensamblaje politiza este proceso de producción y reproducción social para pensar en sus posibilidades de transformación (Puar, Terrorist assemblages 25).

De este modo, los ensamblajes son encuentros complejos, heterogéneos, impuros y estratégicos, con partes intercambiables, que no asumen una esencia anterior a su propia configuración. Tampoco necesitan legitimarse en términos de un exterior constitutivo, evitando la definición en términos binarios contrapuestos, focalizándose en las condiciones y las relaciones a través de las cuales ciertos ordenamientos son posibles. En términos políticos, permiten desarrollar estrategias complementarias a la búsqueda de un posicionamiento táctico para reclamar derechos. Frente a la representación de un sujeto que debe ser reconocido (como por ejemplo «mujer» o «gay»), los ensamblajes apuestan por una política no-representacional que no está ligada a determinadas posiciones de sujeto. Es decir, una política común sin fronteras predefinidas que emerge de la multiplicidad de elementos heterogéneos que favorecen ciertas prácticas contrahegemónicas.

\subsection{El «Mundo Zurdo» de Gloria Anzaldúa}

La obra de Anzaldúa está llena de diversas figuras y conceptos que rompen con las lógicas de representación que hemos venido cuestionando. Anzaldúa se posiciona en los umbrales, simultáneamente dentro y fuera de varios grupos y utiliza precisamente su perspectiva de umbral para desafiar el statu quo. Al hacerlo, reemplaza las políticas reduccionistas de la identidad por nuevas formas de comunidades como el «Mundo zurdo», un lugar visionario donde personas de diferentes orígenes con diversas necesidades y preocupaciones coexisten y trabajan juntas para un cambio revolucionario (Keating, I'm a citizen 60).

Para Anzaldúa el «Mundo Zurdo» o «el mundo de la mano izquierda» representa una perspectiva y espacio altamente creativos, fluidos y de mente abierta. Por lo tanto, la mano izquierda no es un puño sino una mano abierta 
Interseccionalidad, identidad y articulación: hacia una política de la agregación

levantada con otras en la lucha, la celebración y la canción. El concepto de «Mundo Zurdo» es posiblemente el concepto más antiguo de Anzaldúa (Keating, I'm a citizen 63). Comenzó a usar el término a finales de la década de 1970, cuando organizó una serie de lecturas de poesía con ese título en San Francisco, donde se reunieron feministas de color, escritoras estadounidenses del «Tercer Mundo», lesbianas y gays. Desde su diversidad, las personas participantes de la reunión compartieron su crítica a la cultura dominante, sus experiencias personales de discriminación, su interés en temas de justicia social, el rechazo compartido al statu quo y su trabajo como escritores/ as y artistas creativos/as. Varios años después, en la introducción a la tercera sección del libro «Esta puente, mi espalda: Voces de mujeres tercermundistas en los Estados Unidos» y en «La Prieta», Anzaldúa desarrolla la siguiente descripción teórica del «Mundo Zurdo»:

Somos los grupos raros, la gente que no pertenece a ningún sitio, ni al mundo dominante, ni completamente a nuestra propia cultura. Todos juntos abarcamos tantas opresiones. Pero la opresión abrumadora es el hecho colectivo de que no cuadramos, y porque no cuadramos somos una amenaza. No todos tenemos las mismas opresiones, pero tenemos empatía y nos identificamos con las opresiones de cada cual. No tenemos la misma ideología, ni llegamos a soluciones semejantes. Algunos de nosotros somos izquierdistas, algunos somos practicantes de la magia. Algunos de nosotros somos ambos. Pero estas afinidades distintas no se oponen. En el mundo zurdo yo con mis propias afinidades, y mi gente con las suyas, podemos vivir juntos y transformar al planeta. (Moraga y Anzaldua 168)

Son varios los aportes del Mundo Zurdo a la hora de repensar en articulaciones diversas. En primer lugar, tiene como base una epistemología relacional. Anzaldúa visualiza una comunidad compartida por todas las personas a pesar de las diferencias reales entre ellas. Según Anzaldúa «este factor común» va más allá de las identidades basadas en el género, la raza u otros sistemas de diferencia (Keating, I'm a citizen 63). Es decir, es más amplio que cualquier posición social o etiqueta racial. Es importante tener en cuenta que para Anzaldúa este factor de identidad compartida no nos hace idénticos, distinguiendo el término común del de igualdad ya que los puntos en común son siempre heterogéneos y multifacéticos.

En segundo lugar, Anzaldúa ofrece un enfoque diferente, que traspasa la lógica excluyente de la construcción de identidades. Sugiere que las categorías

Feminismo/s 33, junio 2019, pp. 35-63 
Interseccionalidad, identidad y articulación: hacia una política de la agregación

basadas en la identidad se han utilizado y se siguen utilizando para desempoderar y oprimir a las personas en mayor situación de vulnerabilidad. Para ello, propone un «nuevo tribalismo» que trasciende esa lógica excluyente de las categorías. Como ella misma explica:

Muchos de nosotros nos identificamos con grupos y posiciones sociales no limitados a nuestras clasificaciones étnicas, raciales, religiosas, de clase, género o nacionales. Aunque la mayoría de la gente se define por lo que le excluye, nosotros definimos quienes somos por lo que incluimos; lo que llamo un nuevo tribalismo. (Moraga y Anzaldúa 3)

Significativamente, Anzaldúa no descarta la importancia del género, la etnia, raza, la sexualidad, la capacidad y otras categorías relacionadas, sino que sostiene que las categorías convencionales que usamos cotidianamente son demasiado restrictivas y no pueden definirnos adecuadamente (Keating, Entrevistas). Aunque estas marcas son inexactas y obsoletas, los que están en el poder continúan usándolas para destacar y negar a los que son diferentes. La autora nos advierte que cuando nuestras evaluaciones del otro y la otra se basan completamente o principalmente, en sus apariencias físicas y posiciones sociales, hacemos suposiciones sesgadas e imprecisas sobre su visión del mundo, su perspectiva política, etc y esto nos cierra innecesariamente a posibles alianzas. Como afirma Anzaldúa:

Por la actitud políticamente correcta, dejamos que el color, la clase y el género nos separen de aquellos que serían espíritus afines. Así, las paredes se hacen más altas, los abismos entre nosotros se ensanchan y los silencios son más profundos. (Moraga y Anzaldua 296)

Por lo tanto, más que una constitución a partir de la exclusión de los diferentes, la autora propone una constitución en la relación con lo diferente, es decir, la unión a partir del rechazo al statu quo ya que por ser los denominados desviados de la cultura dominante, los habitantes del «Mundo Zurdo» utilizan su propio sentido de la diferencia para forjar nuevas alianzas.

En la misma dirección, Audre Lorde reivindica las políticas de identidad a la vez que las transforma (Ilmonen 11). Para ello, muta la diferencia esencialista de las políticas de identidad en un esfuerzo por la coalición a través de los sistemas de dominación. Ella es la «hermana» pero también la «extranjera», incidiendo en cómo ocupamos posiciones de subjetividad fluidas. Lorde

Feminismo/s 33, junio 2019, pp. 35-63 
Interseccionalidad, identidad y articulación: hacia una política de la agregación

reclama tanto un «yo» como un «nosotros/as» fragmentado y nos recuerda cómo dentro de nuestro «yo» también habita la otredad (Ilmonen 16).

\subsection{La política de lo múltiple de Alejandra Castillo}

La autora chilena Alejandra Castillo indaga en nuevas figuraciones feministas basadas en los signos de polémica y desacuerdo sobre los que se ha escrito y desarrollado el feminismo. Entiende el feminismo como una interrogación constante al modo político y cultural existente, promoviendo otras formas para pensar la política y la cultura: «el feminismo es una palabra doble que interroga sin cesar a las mujeres y a la política» (Castillo, Ars Disyecta 17). Propone, para ello, la figuración de «la política de lo múltiple» (Castillo, Ars Disyecta 11). El hilo conductor que recorre todo su trabajo son las encerronas paradojales que genera la encarnación de las mujeres en la política. Para la autora, es precisamente:

La imposibilidad de un universal que contemple a las mujeres, de una política que las nombre lo que hace de sus políticas una suerte de anticipación de lo que aún no ha advenido, una «realidad utópica» que anida en la contradicción de hundir sus raíces en el presente a la vez que nombra lo que podrá ser. (Rodríguez 258)

De acuerdo a Castillo (Ars Disyecta 11-20), la política de las mujeres emerge precisamente en la polémica, en la crisis del sentido común compartido. El feminismo es el nombre de una política de mujeres que se caracteriza por el complejo juego entre lo excluido e incluido, entre lo particular y universal. La lógica de movimiento de esta política se organiza desde los márgenes hacia el centro con el objetivo de transformar y reinventar la cultura. Gracias a este ejercicio de reinvención y transformación la política feminista puede caracterizarse bajo la forma polémica de la ilimitación, de ser capaz de desanudar y reanudar el juego de las identificaciones y las identidades sociales (Castillo, Ars Disyecta 12-13).

Al conceptualizar el feminismo como una política de lo múltiple, dos tipos de prácticas son posibles, que por lo general suelen ir en sentido contrario: (i) la política de la acción afirmativa y (ii) la política de la interrupción; la primera orientada hacia la presencia igualitaria de las mujeres en el espacio público y la segunda enfocada en desmontar el discurso patriarcal. Por medio 
Interseccionalidad, identidad y articulación: hacia una política de la agregación

de las políticas de la interrupción del sentido común, el feminismo cuestiona el modo moderno de la política. Para Castillo:

No se puede ser feminista sólo habitando en los márgenes: habitando la tranquilidad del margen de la historia (en la escritura de la 'otra historia'); habitando en los márgenes de la lengua (en la audacia de la creación de otras hablas, casi siempre de los sentimientos); habitando, por último, en los márgenes del poder (en la creencia de políticas de la diferencia). (Nudos feministas 23)

En este sentido, las políticas de la interrupción son un juego complejo entre lo universal y lo particular. Retomando la metáfora de los nudos feministas de Julieta Kirkwood (Castillo, Julieta Kirkwood 59), la autora urge a reinventar la política como un todo, y por eso no puede ser una política de interés, sino un proyecto de transformación social. Por ello, Castillo (Julieta Kirkwood 52) al igual que Anzaldúa, apuesta por un feminismo que trabaje en la zona fronteriza del pensamiento, reemplazando las grandes teorías y sospechando continuamente de los universales.

Por lo tanto, en la figuración de la política de lo múltiple, los nudos feministas están presentes cuando surgen interacciones entre distintas mujeres, entre diferentes sujetos políticos feministas, con intereses, necesidades y proyecciones diversas. Esta figuración ofrece dos aportes prometedores. Por un lado, rompe con la idea universal del feminismo como «comunidad de mujeres». Reconceptualiza la relación entre comunidad e individuo, ya que para Castillo «no es la comunidad la que comprende dentro de sí al individuo, sino es el propio individuo el que lleva dentro de sí una comunidad» (El feminismo no es humanismo 20). Propone un rebasamiento crítico del sujeto político del feminismo, donde «los sueños de identidad no son prerrequisitos para la participación política» (Butler 22) y tampoco para la construcción de figuraciones feministas potentes, sino que deben ser comprendidos como una posibilidad de fuga que permite descentrar nuestros lugares más cómodos y problematizar continuamente las posiciones de sujeto que les otorgan sentido. Es por ello que el descentramiento del sujeto se convierte en un horizonte primordial.

Por otro lado, alejándose de las corrientes utilitarias que consideran que el feminismo promueve la idea del «individuo» de la tradición liberal, la política de lo múltiple busca entonces la transformación de la política moderna 
Interseccionalidad, identidad y articulación: hacia una política de la agregación

y no su adecuación. Es una apuesta por un feminismo que no busca una adaptación a la sociedad patriarcal, sino que aspira a la transformación total de las relaciones sociales. El feminismo no es una reificación de la identidad «mujer», no es un humanismo, es ante todo una práctica deslocalizadora del sujeto (Castillo, El feminismo no es humanismo 21).

Por tanto, la «política de lo múltiple» desplaza el sintagma de la «comunidad de mujeres» cuestionando la propia nominación identitaria de «mujer» en tanto «unidad», «identidad»y «naturaleza», para reivindicar las figuras de la alteridad, lo fronterizo, lo múltiple o lo nómada. De hecho, la figura de lo múltiple no pretende ser una reflexión sobre la política, sino más bien un razonamiento sobre sus límites y sus pliegues (Castillo, Lo Humano, La Violencia y Las Mujeres 39).

De esta manera, la operatividad política del feminismo dependerá de su capacidad de construir alianzas a través de las diferencias, ya que atrincherarse en la diferencia oculta la apuesta por lo común y nos excluye del diálogo, afianzando la fractura entre las diversas luchas racista, feminista, ecologista, etc. Cada una de las luchas es una parcialidad que no nos representa de manera holística. Como señala Maalouf, «la identidad no es una yuxtaposición de pertenencias autónomas, es un dibujo en una piel tirante, basta con tocar una sola de esas pertenencias para que vibre la persona entera» (34).

Reconociendo la capacidad que el capitalismo tiene de cooptar las diferencias, de apoderarse de ellas para luego devolverlas vaciadas y homogeneizadas, la política de lo múltiple pretende subvertir esos modos de vida potenciando las diferencias y la singularidad, pero sin abandonar la lucha por un feminismo coalicional. Para ello, como señala Castillo, la política de lo múltiple se ubica en una «realidad utópica» que no tiene tiempo para esperar a su realización en un futuro lejano, sino que, por el contrario, y paradójicamente, busca realizarse en el propio gesto de su enunciación y de nominación feminista (Nudos Feministas 24-25).

En definitiva, estas figuraciones de reinvención de lo humano pretenden salir de los binarismos con los que la política moderna tradicionalmente piensa y construye la identidad. La política de lo múltiple busca en la multiplicidad y en la simultaneidad «humanizar paradójicamente la humanidad» (Castillo, Kirkwood 121). En ese sentido, Donna Haraway considera que pensar lo humano más allá de lo identitario debe dar lugar a nuevas formas,

Feminismo/s 33, junio 2019, pp. 35-63 
Interseccionalidad, identidad y articulación: hacia una política de la agregación

pero que, sin embargo, al mismo tiempo, necesitamos figuras feministas de la humanidad: «La humanidad feminista debe, de algún modo, resistir tanto a la representación como a la figuración literal y aún irrumpir con nuevos y poderosos tropos, nuevas figuras de habla, nuevos giros de posibilidad histórica» (147).

\section{CONSIDERACIONES FINALES}

La centralidad del discurso identitario de las sociedades postfordistas, que priorizaron las reivindicaciones de reconocimiento frente a las de distribución, ha sido ampliamente cuestionado por perspectiva críticas que cuestionan la identidad como prerrequisito para la participación política y abogan por la multiplicidad de posiciones identitarias (Butler 22).

Teniendo en cuenta el actual contexto de crisis sistémica, retroceso de derechos sociales básicos conquistados y auge de los discursos y gobiernos autoritarios, racistas, sexistas y homófobos, en este artículo hemos pretendido analizar en qué medida la interseccionalidad puede ser una herramienta para la repolitización, la transformación social y la articulación de movimientos sociales en base a la enunciación de las diferencias como elemento fundante y punto de arranque. Desplegamos a continuación las principales reflexiones y conclusiones extraídas.

En primer lugar, la teoría de la interseccionalidad ofrece una forma de mediar entre la tensión de reafirmar una identidad múltiple y la necesidad de desarrollar políticas identitarias cuestionando la homogeneización de las categorías identitarias y visibilizando la multiplicidad de los ejes de opresión en el seno de una determinada configuración identitaria. Sin embargo, el potencial crítico de la interseccionalidad como epistemología situada se ha visto condicionado por algunas interpretaciones y usos que vacían su carácter político a través de: a) la reproducción de las dinámicas de esencialización y homogeneización de las política de identidad; (b) la reificación de categorías herméticas ligadas a sistemas binarios y dicotómicos de orden social (i.e.mujer/hombre, negra/blanca) (c) un fetichismo por las diferencias individuales con el riesgo de ser captadas por la compleja industria académica y el multiculturalismo neoliberal. 
Interseccionalidad, identidad y articulación: hacia una política de la agregación

En segundo lugar, es necesario romper con la fragmentación de la lógica identitaria que sigue persistiendo en las políticas públicas, la participación política y en la construcción de imaginarios comunes feministas. La identidad debe ser comprendida como una posibilidad de fuga y, por tanto, como un lugar de descentramiento de nuestros lugares más cómodos, problematizando continuamente las posiciones de sujeto que le dan sentido. De ahí la necesidad metodológica, teórica y política de desplazar la perspectiva identitaria de la noción de interseccionalidad.

Por último, a la hora de pensar en articulaciones urge transitar de las políticas de identidad que consideran la emancipación a partir de la diferencia constitutiva respecto al otro/otra, hacia una política de la agregación donde la emancipación surge a partir de diferencias contingentes que reconocen la articulación con posiciones antagónicas. En ese sentido, la cuestión primordial de una política democrática no reside en llegar a un consenso sin exclusión, es decir a un «nosotras» que no tuviera un «ellas» como correlato, sino establecer una diferenciación que resulte compatible con el pluralismo. Para ello, las figuraciones de los Ensamblajes, el Mundo Zurdo y la Política de lo Múltiple nos ofrecen imaginarios posibles para salir de los binarismos con las que tradicionalmente se construye la identidad y buscar situaciones compartidas sin negar las diferencias y sin abandonar la singularidad y la multiplicidad existentes.

\section{REFERENCIAS BIBLIOGRÁFICAS}

Afroféminas. «Por qué Afroféminas no se suma a la Huelga Feminista». Afroféminas, 2018. 30 mayo 2019. <https://afrofeminas.com/2018/03/05/ porque-afrofeminas-no-se-suma-a-la-huelga-feminista/>

Anthias, Floya, and Nira Yuval-Davis. «Contextualizing Feminism: Gender, Ethnic and Class Divisions». Feminist Review 15 (1983): 62-75. doi: 10.2307/1394792.

Bolsonaro, Jair. «A Íntegra Do Discurso de Jair Bolsonaro Na Posse No Congresso.» HuffPost Brasil, 2019. 30 junio 2019. <_https://www.huffpostbrasil. com/2019/01/01/a-integra-do-discurso-de-jair-bolsonaro-na-posse-no-congresso_a_23631336/? guccounter=1\&guce_referrer_us=aHR0cHM6Ly9kdWNrZHVja2dvLmNvbS8\&guce_referrer_cs=zy6u6LiaOpCcJxgaJYz_QQ >

Feminismo/s 33, junio 2019, pp. 35-63 
Interseccionalidad, identidad y articulación: hacia una política de la agregación

Brah, Avtar. Cartographies of Diaspora: Contesting Identities. New York: Routledge, 1996. https://www.questia.com/library/108874647/cartographiesof-diaspora-contesting-identities.

Brasilia Agencias. «Estallaron Los Memes Contra La Ministra de Jair Bolsonaro Que Habló de Una 'Nueva Era' En Brasil». Clarín, 1 May 2019. <_https://www. msn.com/es-us/noticias/mundo/estallaron-los-memes-contra-la-ministra-dejair-bolsonaro-que-habl\%C3\%B3-de-una-nueva-era-en-brasil/ar-BBROURt > Breines, Wini. The Trouble between Us: An Uneasy History of White and Black Women in the Feminist Movement. Oxford University Press, 2006.

Butler, Judith. Deshacer el género. México DF: Paidós, 2006.

Carastathis, Anna. «The Concept of Intersectionality in Feminist Theory». Philosophy Compass 9. 5 (2014): 304-14. doi: 10.1111/phc3.12129.

Castillo, Alejandra. «Ars Disyecta». Aisthesis 51 (2012): 11-20. doi: 10.4067/ S0718-71812012000100001.

-. «El feminismo no es humanismo». Por un feminismo sin mujeres. Eds. Coordinadora Universitaria por la Diversidad Sexual. Santiago de Chile: Territorios Sexuales Ediciones, 2011.13-21. http://www.bibliotecafragmentada.org/wp-content/uploads/2011/12/La-mujer-como-piedra-de-tope-Unamirada-frente-al-fracaso-del-feminismo.pdf

-. Julieta Kirkwood: Políticas Del Nombre Propio. Santiago de Chile: Palinodia, 2007.

—. «Lo Humano, La Violencia y Las Mujeres». Archivos: Revista de Filosofía 4 (2009): 31-40.

- Nudos feministas. Política, Filosofía y Democracia. Santiago de Chile: Palinodia, 2011.

Collins, Patricia Hill. Black Feminist Thought: Knowledge, Consciousness, and the Politics of Empowerment. New York: Routledge, 2000.

- - "The Difference That Power Makes: Intersectionality and Participatory Democracy». Investigaciones Feministas 8.1 (2017): 19-39. doi: 10.5209/ INFE.54888.

Coll-Planas, Gerard, y Marta Cruells. «La puesta en práctica de la interseccionalidad política: el caso de las políticas LGTB en Cataluña». Revista Española de Ciencia Política 31 (2013): 153-172. http://recp.es/index.php/ recp/article/ view/325/269. 
Interseccionalidad, identidad y articulación: hacia una política de la agregación

Combahee River Collective. «Un manifiesto feminista negro». Intersecciones. Cuerpos y Sexualidades en la Encrucijada. Ed. Lucas Platero. Barcelona: Bellaterra, 2012. 75-86.

Crenshaw, Kimberlé. «Demarginalizing the Intersection of Race and Sex: A Black Feminist Critique of Antidiscrimination Doctrine, Feminist Theory and Antiracist Politics». University of Chicago Legal Forum 1989.8 (1989): 139-167.

-. «Mapping the Margins: Intersectionality, Identity Politics, and Violence against Women of Color». Stanford Law Review 43.6 (1991): 1241-1299. doi: 10.2307/1229039.

Davis, Kathy. «Intersectionality as Buzzword: A Sociology of Science Perspective on What Makes a Feminist Theory Successful». Feminist Theory 9.1 (2008) : 67-85. doi: 10.1177/1464700108086364.

Deleuze, Gilles y Claire Parnet. Dialogues. Columbia University Press, 1987.

Deleuze, Gilles y Félix Guattari. A Thousand Plateaus: Capitalism and Schizophrenia. Translated by Brian Massumi, Continuum, 2008.

Epstein, Steven G. «Gay Politics, Ethnic Identity: The Limits of Social Constructionism». Socialist Review 93 (1987): 9-54, https://www.scholars.northwestern.edu/en/publications/gay-politics-ethnic-identity-thelimits-of-social-constructionism-2.

Falcón, Lidia. «Las últimas perversiones del feminismo». Público, 2017.

30 mayo 2019. <https://blogs.publico.es/lidia-falcon/2017/03/08/ las-ultimas-perversiones-del-feminismo/>

Fraser, Nancy. Fortunes of Feminism: From State-managed Capitalism to Neoliberal Crisis. London: Verso Books, 2013.

Gandarias Goikoetxea, Itziar. «¿Un neologismo a la moda?: Repensar la interseccionalidad como herramienta para la articulación política feminista». Investigaciones Feministas 8.1 (2017): 73-93. doi: 10.5209/INFE.54498.

Gumbs, Alexis Pauline. «Prophecy in the Present Tense: Harriet Tubman, the Combahee Pilgrimage, and Dreams Coming True». Meridians: Feminism, Race, Transnationalism 12.2 (2014): 142-52. https://muse.jhu.edu/article/558788. Hall, Stuart. «Introduction: Who Needs 'Identity'?». Questions of Cultural Identity. Eds. Stuart Hall and Paul du Gay. London: Sage Publications Ltd, 1996. 1-17. doi: $10.4135 / 9781446221907$. 
Interseccionalidad, identidad y articulación: hacia una política de la agregación

Hancock, Ange-Marie. «When Multiplication Doesn't Equal Quick Addition: Examining Intersectionality as a Research Paradigm». Perspectives on Politics 5. 1 (2007): 63-79. doi: 10.1017/S1537592707070065.

Haraway, Donna. «La Promesa de Los Monstruos». Política y Sociedad 30 (1999): 121-163.

Hardt Michael, y Antonio Negri. Multitud: Guerra y Democracia En La Era Del Imperio. Barcelona: Editorial Debate, 2004.

Ilmonen, Kaisa. «Identity politics revisited: On Audre Lorde, intersectionality, and mobilizing writing styles». European Journal of Women's Studies 26.1 (2017): 7-22. doi: 10.1177/1350506817702410.

Ivaldi, Gilles. «A New Course for the French Radical Right? The Front National and 'de-Demonisation'». Radical Right-Wing Populist Parties in Western Europe: Into the Mainstream?. Eds. Tjitske Akkerman, Sarah L. De Lange, and Matthijs Rooduijn. New York: Routledge, 2016. 225-246.

Keating, Ana Louise. «I'm a Citizen of the Universe': Gloria Anzaldúa's Spiritual Activism as Catalyst for Social Change». Feminist Studies 34. 1/2 (2008): 53-69.

-. Gloria E. Anzaldúa: Interviews/Entrevistas. New York and London: Routledge, 2000.

Law, John. After method: Mess in social science research. New York: Routledge, 2004.

Lilla, Mark. The Once and Future Liberal: After Identity Politics. New York, NY: Harper Collins, 2017.

Lorde, Audre. Sister Outsider: Essays and Speeches. Freedom: The Crossing Press, 1984.

Maalouf, Amin. Identidades asesinas. Madrid: Alianza, 1999.

Mohanty, Chandra Talpade. «On race and voice: Challenges for liberal education in the 1990s». Cultural Critique 14. (1989): 179-208.

-. «Under Western eyes: Feminist scholarship and colonial discourses». Feminist Review 30. 1(1988): 61-88.

Moraga, Cherríe, y Gloria Anzaldúa. This Bridge Called My Back: writings by radical women of color. New York: Kitchen Table: Women of Color Press, 1981.

Nail, Thomas. «What Is an Assemblage?» SubStance 46.1 (2017): 21-37. https:// muse.jhu.edu/article/650026.

Nash, Jennifer C. «Re-Thinking Intersectionality». Feminist Review 89 (2008): $1-15$. 
Interseccionalidad, identidad y articulación: hacia una política de la agregación

Nayak, Suryia, Marisela Montenegro, y Joan Pujol. «Conclusion: Contextual Intersectionality: A Conversation». Intersectionality in Social Work: Activism and Practice in Context. Eds. Suryia Nayak and Rachel Robbins. New York: Routledge, 2018. 230-250.

Nayak, Suryia, y Rachel Robbins. «Introduction». Intersectionality in Social Work: Activism and Practice in Context. Eds. Suryia Nayak and Rachel Robbins. New York: Routledge, 2018. 1-5.

New York Times. «Transcript: Donald Trump's Victory Speech». The New York Times, 2016, 30 junio 2019. <https://www.nytimes.com/2016/11/10/us/politics/trump-speech-transcript.html>.

Platero Méndez, Lucas (Raquel). «¿Son las políticas de igualdad de género permeables a los debates sobre la interseccionalidad? Una reflexión a partir del caso español.» Revista del CLAD Reforma y Democracia 52 (2012): 135-172. http://www.redalyc.org/resumen.oa?id=357533684005.

Polanyi, Karl. La gran transformación. México, Juan Pablos Editor, 1992.

Puar, Jasbir K. "I Would Rather Be a Cyborg than a Goddess': BecomingIntersectional in Assemblage Theory». Philosophia: A Journal of Feminist Continental Philosophy 2. 1 (2013): 49-66.

-. Terrorist assemblages: Homonationalism in queer times. NC: Duke University Press, 2017.

Rodríguez, Rosana. «Nudos Feministas. Política, Filosofía, Democracia». Nomadias 14 (2011): 257-261.

Romero Bachiller, Carmen, y Marisela Montenegro Martínez. «Políticas públicas para la gestión de la diversidad sexual y de género: un análisis interseccional». Psicoperspectivas. Individuo y Sociedad 17.1 (2018): 1-14. doi: 10.5027/ psicoperspectivas-Voll7-Issuel-fulltext-1211.

Romero Bachiller, Carmen. «Los desplazamientos de la «raza»: de una invención política y la materialidad de sus efectos». Política y Sociedad 40.1 (2003): 111-128.

Spade, Dean. «Intersectional Resistance and Law Reform». Signs: Journal of Women in Culture and Society 38.4 (2013): 1031-1055. doi: 10.1086/669574. Taylor, Keeanga-Yamahtta. «Introduction». How We Get Free: Black Feminism and the Combahee River Collective. Ed. Keeanga-Yamahtta Taylor. Chicago: Haymarket Books, 2017. 1-14. https://alexy.asian.lsa.umich.edu/courses/readings/Taylor_How\%20We\%20Got\%20Free.pdf.

Feminismo/s 33, junio 2019, pp. 35-63 
Truth, Sojourney. «Ain't I a woman? (speech)». Feminism: The essential historical writings. Ed. M. Schneir. 128 [131]. New York: Random House, 1851. 463-464.

Unzueta, María Angeles Barrere. «Problemas del derecho antidiscriminatorio: subordinación versus discriminación y acción positiva versus igualdad de oportunidades.» Revista Vasca de Administración Pública. Herri-Arduralaritzako Euskal Aldizkaria 60 (2001): 145-166.

Vox. Programa Electoral de VOX: 100 Medidas Para La España Viva, 2018. 30 mayo 2019. <https://www.voxespana.es/biblioteca/espana/2018m/gal_ c2d72e181103013447.pdf>

Walters, Suzanna Danuta. «In Defense of Identity Politics». Signs: Journal of Women in Culture and Society 43.2 (2017): 473-88. doi: 10.1086/693557.

Yuval-Davis, Nira. «Situated Intersectionality and the Meanings of Culture» Consello da Cultura Galega (2017) 1-10. 30 mayo 2019. <http://consellodacultura.gal/ mediateca/extras/Texto_Nira_maquetado.pdf> 